\title{
Solar radiative heating in first-year sea ice
}

\author{
M. J. MaGuinness, ${ }^{1}$ K. A. Landman, ${ }^{2}$ H. J. Trodahl, ${ }^{3}$ A. E. Pantoja ${ }^{3}$ \\ ${ }^{1}$ School of Mathematical and Computing Science, Victoria University of Wellington, P.O. Box 600, Wellington, New Zealand \\ ${ }^{2}$ Department of Mathematics and Statistics, University of Melbourne, Melbourne, Australia \\ ${ }^{3}$ School of Chemical and Physical Sciences, Victoria University of Wellington, Wellington, New Zealand
}

\begin{abstract}
Temperature measurements taken in young, landfast Antarctic sea ice show daily oscillations consistent with heating by solar radiation. We present and solve a heat conduction model for the temperature with a non-linear thermal capacity and a distributed source term for solar power absorption based on Monte Carlo scattering simulations of penetrating photons. We observe two characteristic modes for solar heating in sea ice, one dominated by travelling thermal waves or conduction in the upper half, and the other dominated by in-place solar heating in the lower half. We note that deep thermal responses to solar radiation are larger by a factor of $\sim 10$ than predicted by scattering measurements, due possibly to the presence of algae and/or dissolved organic material.
\end{abstract}

\section{INTRODUGTION}

Sea ice in the Arctic and the Antarctic plays a well-recognized part in climate and ocean modelling. One aspect of some interest is the thermal response of sea ice to solar radiation (e.g. Flato and Brown, 1996; Key and others, 1996; Hanesiak and others, 1999). In temperature measurements taken to calculate the thermal conductivity we have noted (McGuinness and others, 1998; Trodahl and others, 2000) daily oscillations consistent with solar heating. The accuracy and resolution of these measurements provide a direct measurement of the thermal effect of solar radiation. A preliminary analysis of the observed thermal response to solar heating appears in Trodahl and others (2000), and we seek to extend this work here. In particular, we seek to analyze more accurately the conductive and volumetric heating effects.

In this paper we report on the temperature data gathered in McMurdo Sound, Antarctica, we describe the Monte Carlo scattering simulations performed to calculate the absorbed solar power as a function of depth in sea ice, we outline and solve a mathematical model for the diffusion of heat in sea ice that incorporates solar heating as a distributed source term, and finally we compare and discuss the results.

\section{TEMPERATURE DATA}

Thermistor arrays frozen into first-year, landfast sea ice in McMurdo Sound in 1996 and 1999 yield hourly temperature measurements at 20 depths spaced $0.1 \mathrm{~m}$ apart during winter and spring. The chosen locations, one about $1 \mathrm{~km}$ off Arrival Heights, and the other in the lee of Big Razorback Island off Cape Evans, had little snow accumulation. The ice in both locations grows to a thickness of $>2 \mathrm{~m}$ in winter.

Recorded temperatures are plotted against time in Figures 1 and 2. Each line of data corresponds to one thermistor, so that the higher temperatures correspond to the deeper (warmer) thermistors. The Omega 44031 thermistors used are calibrated and uniform to $0.1^{\circ} \mathrm{C}$, but their potential resolution is $\sim 0.001^{\circ} \mathrm{C}$. Actual uncertainty is in practice $\sim 0.01^{\circ} \mathrm{C}$.
Of some concern is the possibility that the observed temperature oscillations are due to the sun directly heating the thermistor string, rather than to variations in ice temperature. The direct heating of the thermistors by sunlight has been modelled in Trodahl and others (2000) and was found to be negligibly small in amplitude. Furthermore it would be in phase with the sun, and this is not observed in our data.

The temperature data show changes forced by variations in air temperature, and also during some periods in spring a smaller, faster (daily) oscillation can be seen superimposed on the slower changes, which we assume is due to solar forcing. It is this solar forcing which we investigate further in this paper.

There are periods during spring when no solar forcing is visible; we believe this is when cloud obscures the sun.

\section{MONTE GARLO SGATTERING}

The optical energy absorbed per unit volume at any depth $z$ in the ice is given by the product of the average radiance and the absorption coefficient. However, the average radiance is not easily determined within the very successful Monte Carlo models we have developed on the basis of lightspreading measurements. We have thus adapted these models to simulate the absorption process.

The core element in the Monte Carlo models is the tracing of many randomly scattered light paths for rays impinging on the surface. The effects of scattering are modeled by a depth-dependent scattering length (meanfree-path) whose magnitude and depth dependence are chosen to reproduce experimentally-determined beamspreading profiles (Haines and others, 1997). In the present case, where we wish to simulate the heat absorbed in the ice, we follow the paths through many layers of thickness $\Delta z$ centred at depths $z_{i}=\left(i+\frac{1}{2}\right) \Delta z$, and record for each traverse $j$ of a ray through that layer the path length $\ell_{i}$ spent in the layer, binned by the total path $L_{j}$ that the ray has followed before reaching that level. Then the absorbed power per unit volume at that depth due to a flux $F_{\lambda} \Delta \lambda$ falling on 

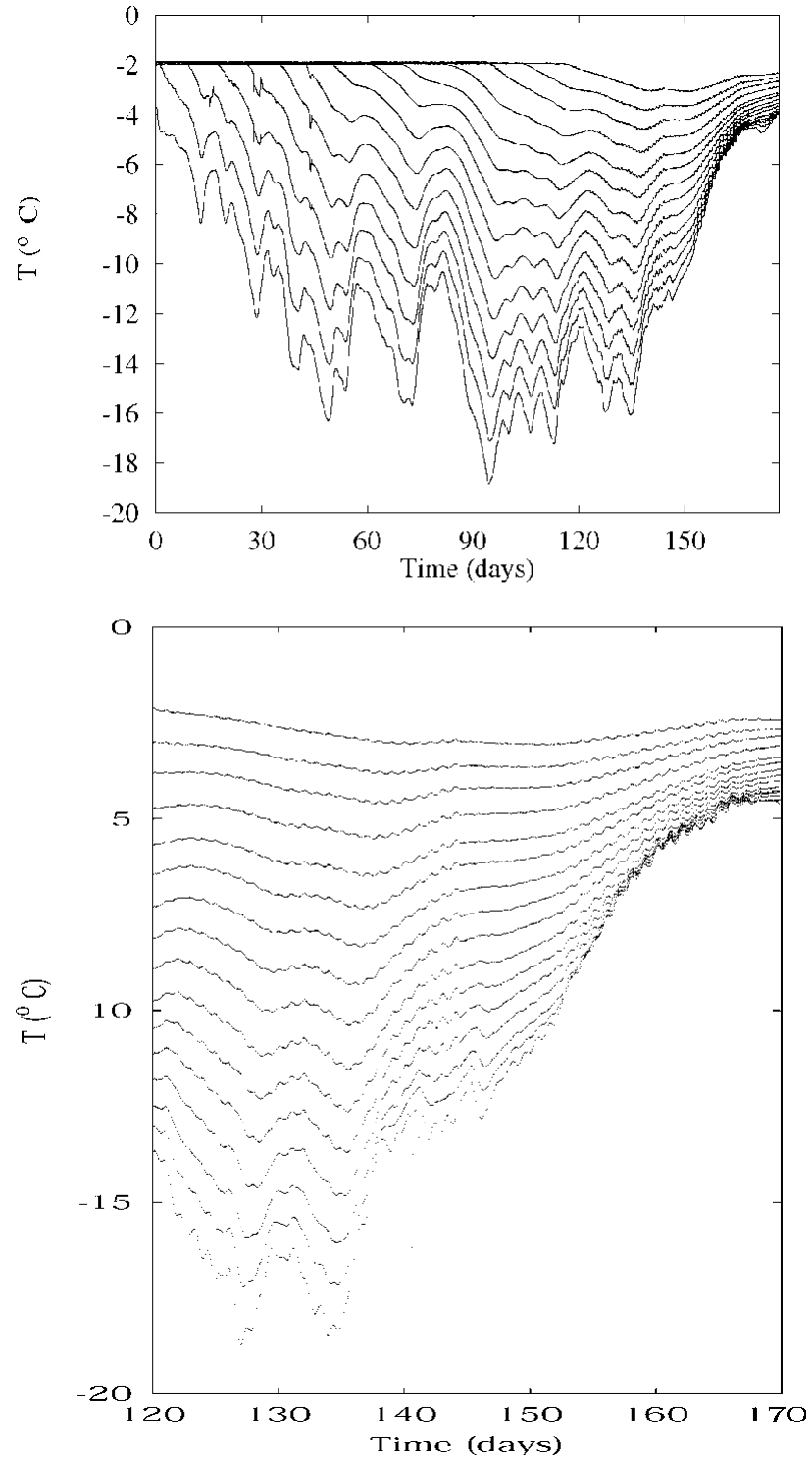

Fig. 1. Temperatures measured in 1996, plotted against time. Each line is the temperature recorded by one thermistor at a constant depth. The upper plot shows temperatures during winter and spring at depths 0.7-2.0 m, with the coldest temperatures being from the $0.7 \mathrm{~m}$ deep thermistor. The lower plot shows more detail during spring, and is for depths $0.5-2.0 \mathrm{~m}$.

Day zero is 12 June.

the surface in wavelength bands $\lambda$ to $\lambda+\Delta \lambda$, and summed over all wavelengths, is

$$
P\left(z_{i}\right)=P_{i}=\frac{\Delta \lambda}{N \Delta z} \sum_{\lambda}\left\{F_{\lambda} \kappa_{\lambda} \sum_{j}\left[\left(\sum \ell_{i}\right)_{j} \mathrm{e}^{-\kappa_{\lambda} L_{j}}\right]\right\}
$$

where $N$ is the number of rays used in the Monte Carlo model, and $\kappa_{\lambda}$ is the absorption coefficient for pure ice in the wavelength band $\lambda$ to $\lambda+\Delta \lambda$. The wavelength range used is $300-1400 \mathrm{~nm}$. The specific models, specified by the depth-dependent scattering length, include three of those determined for McMurdo Sound first-year ice (Haines and others, 1997). We note that they all display a strongly scattering surface layer and a more weakly scattering interior, and these features will be seen below to influence the light-absorption profiles. Such a two-layer structure is

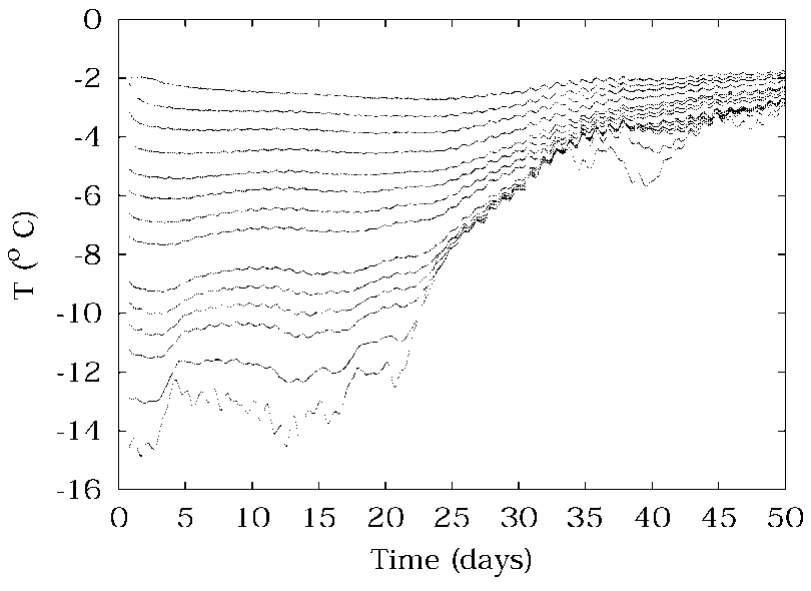

Fig. 2. Temperatures measured in spring 1999, plotted against time, from thermistors at depths $0.4-1.9 \mathrm{~m}$. Data from 0.6 and $1.1 \mathrm{~m}$ are missing, due to faulty thermistors. Day zero is 14 October.

also apparent in studies of Arctic first-year sea ice (e.g. Perovich and others, 1998).

The base ten logarithms of the resulting power densities are plotted against depth in Figure 3. The models used range from the most transparent (curve c), through an intermediate case (curve a), to the most opaque or turbid (curve b) of those developed by Haines and others (1997). The powers are for midday during spring, and are used as an estimate of the peak-to-peak variation of solar power. They are used in the distributed source term in the heat-conduction modelling in the next section, and they are the most important factor in determining the amplitudes of temperature oscillations in the ice due to solar radiation penetration.

\section{MATHEMATICAL MODELLING}

We model the temperature field in the sea ice with a onedimensional heat-diffusion equation. Heating by solar radiation is included as a distributed source term, as

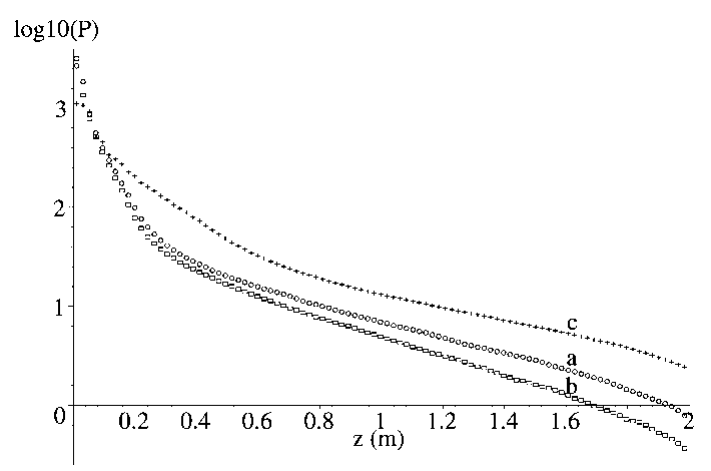

Fig. 3. The base ten logarithm of solar power absorbed per unit volume $\left(\mathrm{f} \mathrm{m}^{-3}\right)$, vs depth in the sea ice, calculated by Monte Carlo scattering, and using scattering lengths fitted to 1986 experiments. The labels $a, b, c$ on the curves correspond to cases $86 \mathrm{~A}, 86 \mathrm{~B}$ and $86 C$, respectively, used by Haines and others (1997). The power is that at midday on a typical spring day in McMurdo Sound. 
calculated in the previous section. The equation describing the conduction of heat in the sea ice is

$$
\frac{\partial \theta}{\partial t}=D(\theta) \frac{\partial^{2} \theta}{\partial z^{2}}+Q(\theta, z)\left(1+A \mathrm{e}^{i \omega t}\right),
$$

where the diffusivity is

$$
D(\theta)=\frac{k}{\rho C(\theta) L^{2}}
$$

and the solar driving term is

$$
Q(\theta, z)=\frac{P(z)}{\rho C(\theta)}
$$

where temperature is the real part of $\theta$ in ${ }^{\circ} \mathrm{C}, \theta$ is a complexvalued function of depth $z$ and time $t, k$ is thermal conductivity, here taken to be $2 \mathrm{~W} \mathrm{~m}^{-1} \mathrm{~K}^{-1}, \rho$ is the density of the sea ice, and $P$ is the solar power absorbed per unit volume $\left(\mathrm{J} \mathrm{m}^{-3}\right)$ of sea ice. A complex-valued $\theta$ is computationally convenient for analyzing oscillations. Depth $z$ is zero at the upper ice/air interface, and has been rescaled to give $z=1$ at the lower ice/water interface, using the factor $L$, the total ice thickness, which is taken to be $L=2$ during spring.

The factor $\left(1+A \mathrm{e}^{i \omega t}\right)$ accounts for the daily variation in amplitude as the sun rises and falls, with frequency $\omega=2 \pi$ $\operatorname{radd}^{-1}$. $A$ has modulus one, which corresponds to the sun just touching the horizon overnight. The argument of $A$ sets the zero of time with respect to the sun's periodic behaviour. $C(\theta)$ is the temperature-dependent heat capacity per unit mass of sea ice $\left(\mathrm{kJ} \mathrm{kg}^{-1}{ }^{\circ} \mathrm{C}^{-1}\right)$, and we use the empirical formula (Schwerdtfeger, 1963; Ono, 1966; Yen, 1981)

$$
\begin{aligned}
C(\theta)= & 2.113-0.0033 S \\
& +(0.0065+0.0008 S) \theta+18.04 S / \theta^{2},
\end{aligned}
$$

where $S$ is the salinity in practical salinity units (psu) of the sea ice, here taken to be 5.5 psu.

The boundary condition used at the sea/ice interface where $z=1$ is to fix the temperature at the value $\theta_{0}=-1.8^{\circ} \mathrm{C}$.

For the boundary condition at the upper ice surface $z=0$, a Newton-type heat loss is used, with the temperature gradient at the surface proportional to the difference between the ice temperature and the air temperature,

$$
\frac{\partial \theta}{\partial z}=\alpha\left(\theta-\theta_{\text {air }}\right) \quad \text { at } z=0
$$

where $\alpha$ is some positive constant. If $\alpha=0$, the ice is perfectly insulated from the air. If $\alpha \rightarrow \infty$, the ice temperature at the surface is equal to the air temperature there. Equation (6) corresponds to the sensible-heat flux, mentioned, for example, in Zeebe and others (1996). Their work suggests the value $\alpha \approx 12$ at average wind speeds in McMurdo Sound.

This boundary condition may appear crude compared, for example, with that of Zeebe and others (1996), but incoming and outgoing radiation effects at the upper surface are already accounted for explicitly by the Monte Carlo simulations through the source term $P(z)$, for wavelengths in the range $300-1400 \mathrm{~nm}$, and hence are not needed in the boundary condition. Radiation at wavelengths of $>1400 \mathrm{~nm}$ is ignored here, because we see very little power in the spectrum of the solar radiation striking the ice surface above $1400 \mathrm{~nm}$. We also ignore latent-heat effects at the ice/air interface. For the Weddell Sea, latent-heat fluxes have been estimated (Eicken, 1992) to be about a quarter of the size of the sensible-heat fluxes. Furthermore, we find in the following sections that the oscil- latory solar forcing solutions are not very sensitive to the boundary condition used at the upper surface.

\section{Asymptotic analysis}

The model equation (2) for temperature is non-linear, and as it stands it is difficult to extract information about solution responses to penetrating solar radiation. However, large temperature variations on time-scales of the order of 1 week or more (slow time), and smaller daily temperature oscillations (fast time), are visible in the temperature data presented in Figures 1 and 2. Hence we will make progress by proceeding to analyze the daily small-scale changes as perturbations on the longer-term trends.

We seek a temperature expansion in the form

$$
\theta=\psi(\tau, z)+\epsilon v(t, \tau, z)+\mathcal{O}\left(\epsilon^{2}\right),
$$

where (following, e.g., Kevorkian and Cole, 1981) we are using a multiple time-scales analysis, with $t$ a fast time, $\epsilon$ a small parameter, and $\tau=\epsilon t$ a slow time-scale. Note that we are explicitly taking the leading behaviour $\psi$ to be slowly varying in time. If we choose $Q$ to also be of size $\epsilon$, we find the background temperature $\psi$ solves the steady-state problem

$$
D(\psi) \frac{\partial^{2} \psi}{\partial z^{2}}=0
$$

which has a solution of the form

$$
\psi(\tau, z)=E(\tau)+F(\tau) z
$$

Substituting this into the boundary conditions then gives

$$
\psi(\tau, z)=\left(\frac{1}{1+\alpha}\right)\left[\theta_{0}+\alpha \theta_{\text {air }}(\tau)+\alpha\left(\theta_{0}-\theta_{\text {air }}(\tau) z\right)\right] .
$$

The temperature at the top of the sea ice $(z=0)$ is

$$
\psi_{\text {top }}=\left(\frac{1}{1+\alpha}\right)\left(\theta_{0}+\alpha \theta_{\text {air }}\right) \text {. }
$$

Note that as $\alpha \rightarrow \infty, \psi_{\text {top }} \rightarrow \theta_{\text {air }}$, as expected since large $\alpha$ corresponds to good thermal contact between ice and air. The leading temperature behaviour $\psi$ is linear in $z$, taking the values $\theta_{0}$ at $z=1$ and $\psi_{\text {top }}$ at $z=0$.

The $\mathcal{O}(\epsilon)$ problem is

$$
\frac{\partial v}{\partial t}-D \frac{\partial^{2} v}{\partial z^{2}}=Q\left(1+A \mathrm{e}^{i \omega t}\right)-\left(\frac{\alpha}{1+\alpha}\right)(1-z) \frac{\mathrm{d} \theta_{\text {air }}(\tau)}{\mathrm{d} \tau}
$$

where $D=D(\psi(\tau, z))$ and $Q=Q(z, \psi(\tau, z))$.

This is a linear partial differential equation for $v$. The righthand side consists of oscillatory and non-oscillatory forcing terms, and we are only interested in the oscillatory behaviour of $v$. Hence we let

$$
v=T(t, \tau, z)+V(z) \mathrm{e}^{i \omega t}
$$

and we want to determine $V(z)$. $|V|$ gives the amplitude and $-\arg (V)$ gives the phase shift of the oscillations in $v$. Substitution into Equation (12) and equating coefficients of $\mathrm{e}^{i \omega t}$ gives

$$
D(\psi) \frac{\mathrm{d}^{2} V}{\mathrm{~d} z^{2}}-i \omega V=-Q(z, \psi) A
$$

which is to be solved for $V$ subject to the boundary conditions $V(1)=0$ and

$$
\frac{\mathrm{d} V}{\mathrm{~d} z}=\alpha V, \quad \text { at } z=0 .
$$

This is a boundary-value problem for a linear inhomogeneous second-order ordinary differential equation with 


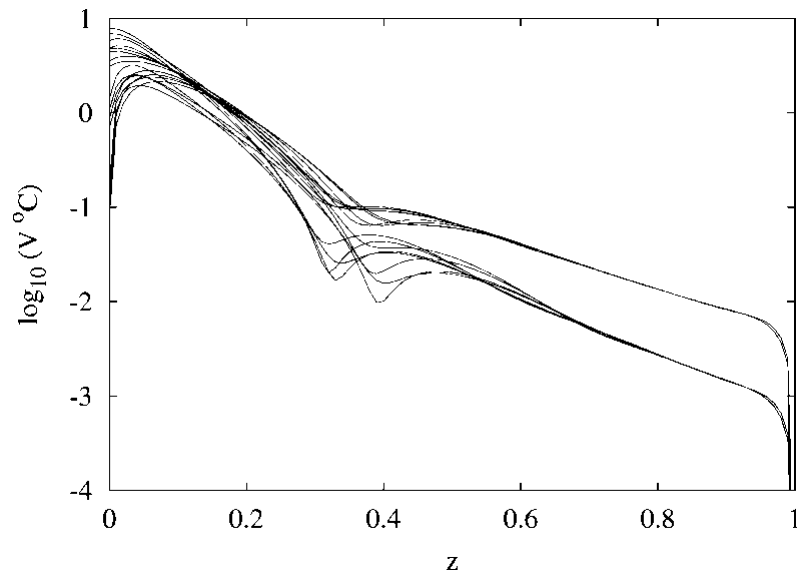

Fig. 4. Amplitudes of temperature oscillations obtained from numerical model solutions. The different curves correspond to different parameter values as explained in the text.

variable coefficients, which cannot be solved by elementary methods. However, numerical solutions will be discussed in the following sections, and asymptotic solutions are the subject of a paper in preparation.

\section{Numerical solutions}

Numerical solutions have been obtained for Equation (14), using Mathematica and a standard numerical procedure called the shooting method (e.g. Press and others, 1986). Plots of numerical solutions appear in Figures 4 and 5. The two major line groupings in the amplitude plot correspond to the two extreme choices of cases $\mathrm{b}$ and $\mathrm{c}$ for $P(z)$. The effect of changing from case $b$ to case $c$ is not as dramatic in the phase curves. The multiple clusters of lines within these two major groupings correspond to different choices of the parameters $\psi_{\text {top }}$ and $\alpha$. Numerical solutions can be seen to be relatively insensitive to these parameters, depending mainly on the scattering properties of the sea ice (i.e. on cases $\mathrm{b}$ and $\mathrm{c}$ ). The values actually used for $\alpha$ are $0,1,10,100$ and 1000. The value 1000 was found to give results indistinguishable from infinity, so the full range of surface boundary conditions are allowed for, from fully insulating

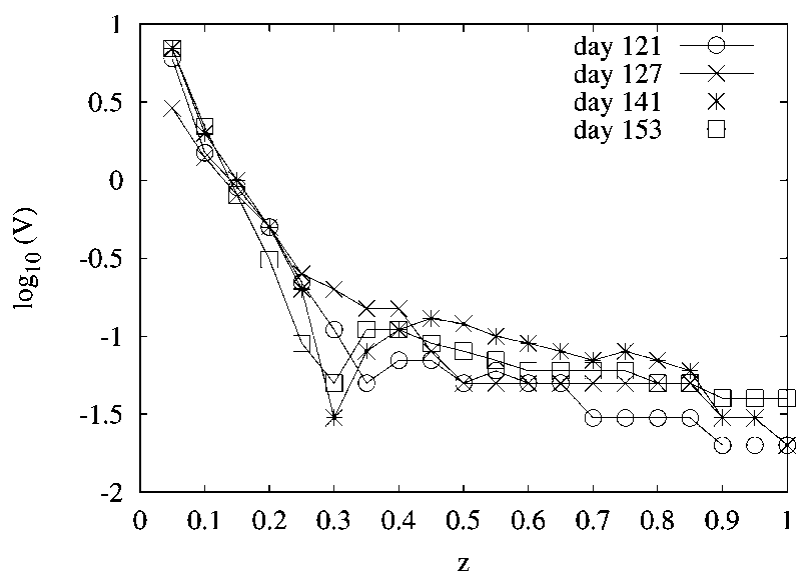

Fig. 6. Amplitudes of temperature oscillations observed in spring 1996. The day number refers to the numbering used in the temperature data (1), and indicates the day near which the data were obtained.

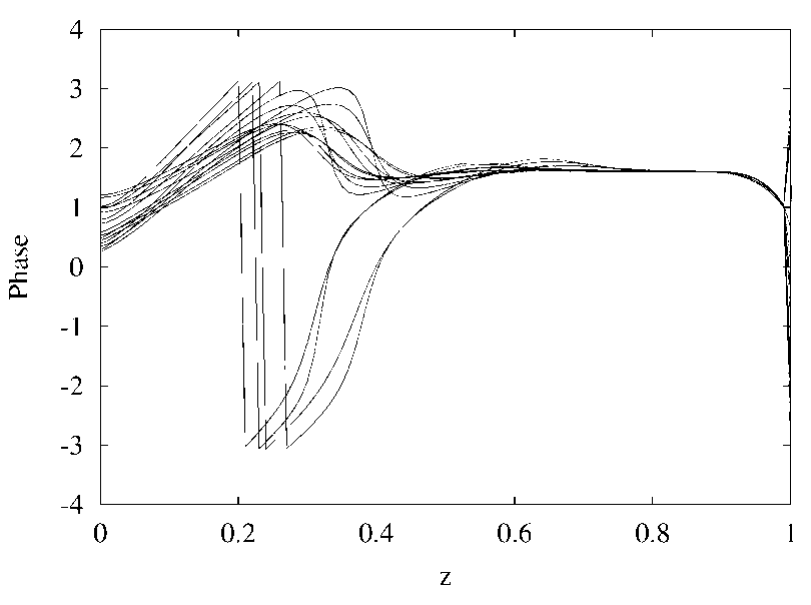

Fig. 5. Phases of temperature oscillations obtained from numerical model solutions. The different curves correspond to different parameter values as explained in the text.

to perfect thermal contact. Values chosen for the surface air temperature $\psi_{\text {top }}$ range from $-5^{\circ}$ to $-20^{\circ} \mathrm{C}$.

Of some interest in the numerical solutions is the appearance of a solid-state greenhouse effect, with the maximum in temperature oscillation amplitude appearing at about $0.1 \mathrm{~m}$ depth beneath the surface of the ice, for values of $\alpha$ that correspond to good thermal contact with the air. This is not observed in the data, since the spacing of the thermistors is not close enough near the ice surface to resolve it.

\section{DATA AND DISGUSSION}

Temperature data from spring of 1996 and of 1999 have been analyzed to obtain the amplitudes and relative phases of those oscillations with a period of 1 day, and the results are displayed in Figures 6-9. Note that the scaled depth $z=1$ corresponds to $2 \mathrm{~m}$.

The phases plotted are relative phases, with deep phases arbitrarily set to zero, and with the allowed range of phase being from zero to $2 \pi$.

A two-layer structure or behaviour is clearly evident in the data, and correlates well with what is observed in the numerical solutions. In a shallow layer we call the conductive region, up to $\sim 0.8 \mathrm{~m}$ deep $(0 \leq z \leq 0.4)$, amplitudes

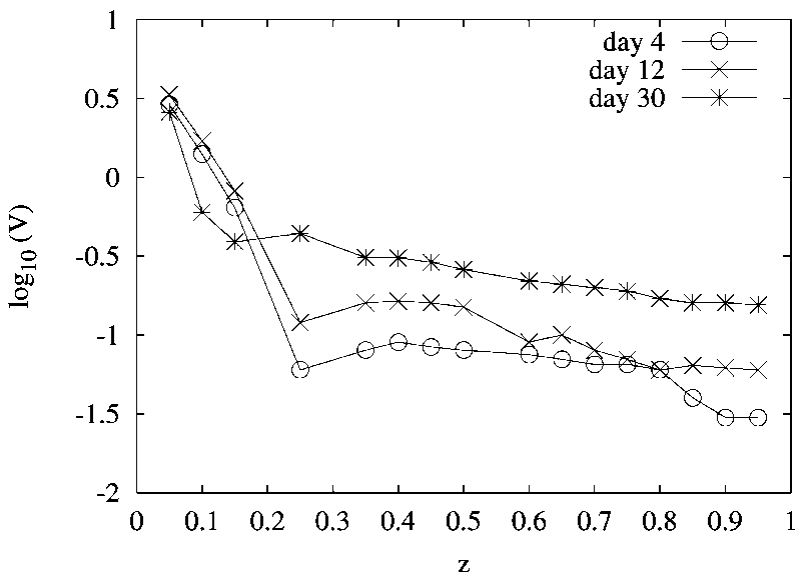

Fig. 7. Amplitudes of temperature oscillations observed in spring 1999. The day number refers to the numbering used in the temperature data (2), and indicates the day near which the data were obtained. 


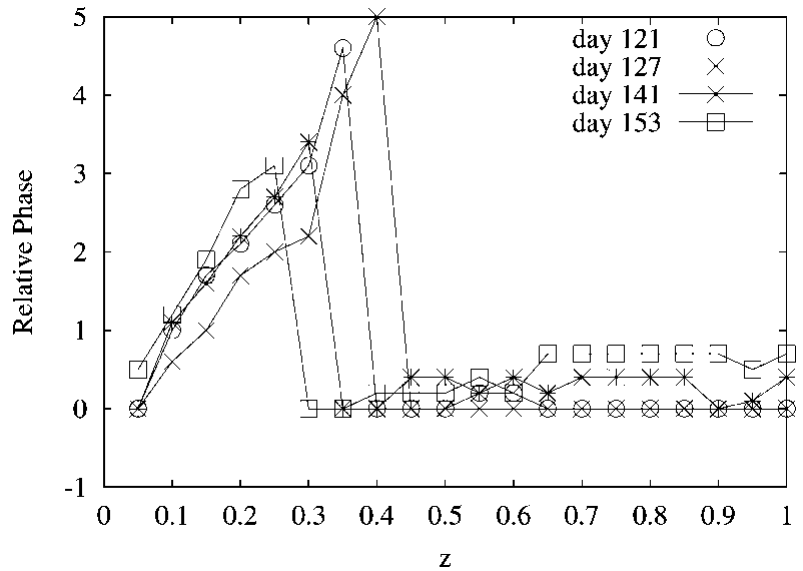

Fig. 8. Relative phases of temperature oscillations observed during spring 1996. The day number refers to the numbering used in the temperature data (1), and indicates the day near which the data were obtained.

decrease most rapidly, and the phase varies approximately linearly with depth, consistent with (damped) travelling waves of constant velocity. Temperature changes in this conductive region are driven by the rapid changes in amplitude associated with rapid changes in the solar driving term $P$.

The fact that much of the incoming solar radiation is absorbed in a shallow layer is well known, and Zeebe and others (1996) use this to justify accommodating the effects of longer wavelengths in their boundary condition at the surface of the sea ice. What is perhaps surprising here is the large thermal footprint of the shallow layer, in that the travelling waves originating there can be seen to penetrate nearly $1 \mathrm{~m}$ into the sea ice before their amplitudes diminish to the size of the deeper layer amplitudes. The size of this footprint is seen to be reduced after warming the ice, when deep amplitudes are increased, particularly in Figure 7.

In the deeper layer, observed amplitudes decrease relatively slowly, and phase is almost constant at $\pi / 2$. This corresponds physically to simple volumetric solar heating with negligible conductive heat flow. We observe that after the ice warms to near $-5^{\circ} \mathrm{C}$, deep amplitudes increase, and the conductive region shrinks in size. This effect is particularly apparent in Figures 7 and 9, with day 30 amplitudes raised

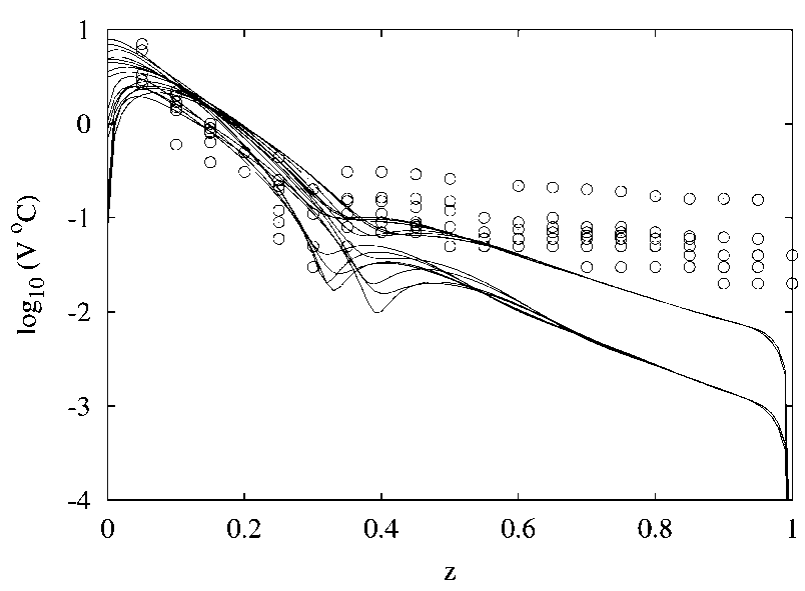

Fig. 10. Predicted and observed values of the amplitude of temperature oscillations, plotted together for comparison. All of the data have been plotted as circles, and the numerical results as solid lines.

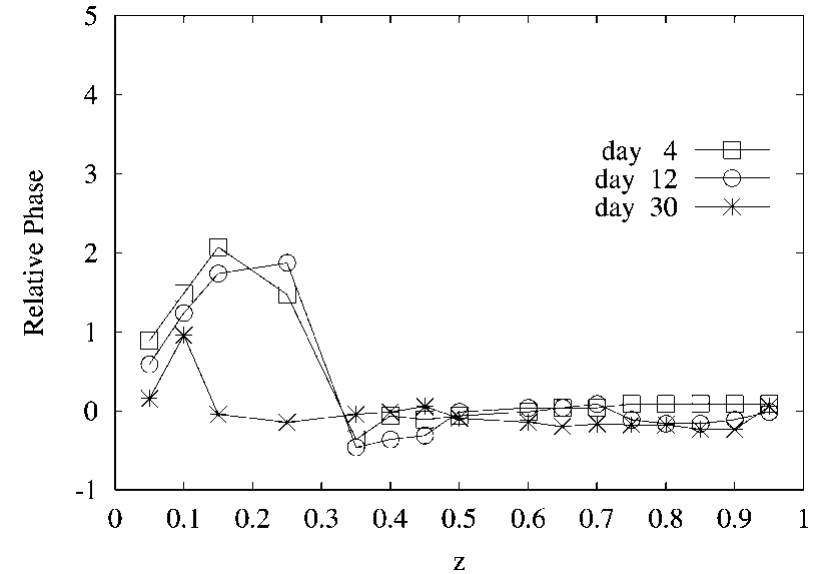

Fig. 9. Relative phases of temperature oscillations observed during spring 1999. The day number refers to the numbering used in the temperature data (2), and indicates the day near which the data were obtained.

at depth compared with the other deep amplitudes, and the conductive region from the phase plot for day 30 noticeably smaller. Note that day 30 was in a period of significant warming for the 1999 data (Fig. 2).

\section{NUMERICAL SOLUTION MATGHES}

In Figures 10 and 11 the numerical solutions from Figures 4 and 5 are plotted together with all of the observed results in Figures 6-9, on the same graph, for comparison purposes. Relative phases obtained from temperature data have had the zero adjusted so that the deep phase is $\pi / 2$. Otherwise no fitting has been done; the numerical results are based entirely on incident solar radiation, model scattering lengths, and the absorption coefficient for pure ice, as discussed above.

Phase matches between data and numerical model solutions are excellent, at all depths. The jumps that can be seen in the phases are due to restricting phase to principal values between $(-\pi, \pi)$.

Amplitudes also match well in the shallow conductive layer, but the deep amplitudes are larger (by a factor of $\sim 10$ ) in the measured data than predicted by the model solutions.

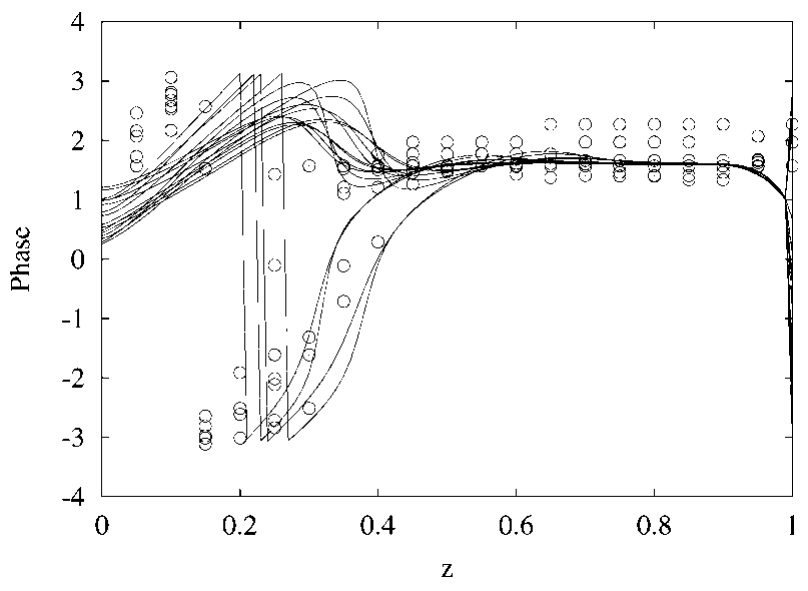

Fig. 11. Predicted and observed values of the phase of temperature oscillations, plotted together for comparison. All of the data have been plotted as circles, and the numerical results as solid lines. 
As noted above, deep amplitudes are largest after the ice has warmed. Key factors that might account for the discrepancy are scattering lengths and absorption coefficients. However, previous studies of the temperature dependence of scattering lengths in sea ice (Haines and others, 1997) have shown that the deep scattering lengths are not perceptibly changed by warming.

This suggests that the anomalously high temperatureoscillation amplitudes observed at depth may be due to the extra absorption attributable to the presence of algae (Zeebe and others, 1996) or dissolved organic material in the deeper layer (Perovich and others, 1998), since the Monte Carlo simulations we conducted assumed that attenuation of photons between scattering centres was due only to pure ice. The discrepancy between data and numerical solution amplitudes at depth shows as a deviation in slope on the semilog plot, suggestive of a distributed effect rather than any localized concentration of algae in strata. Grenfell (1991) notes that absorption by algae can reduce transmitted radiation by more than a factor of ten at certain visible wavelengths. Perovich and others (1998) discuss possible mechanisms that could enhance entrapment of organic matter during the slow growth of congelation ice that typifies the deeper sea-ice layer, and suggest that an alternative ice-growth mechanism such as a sub-ice frazil layer or buoyant anchor ice could bring particles of organic matter to the ice. We would add to this list the possibility of platelet ice also bringing organic matter to be incorporated into the sea-ice matrix (e.g. Crocker and Wadhams, 1989; Jeffries and others, 1993; Gow and others, 1998; Smith and others, 1999).

The data presented here represent a direct measurement of the effect of solar radiation penetration in sea ice, and may serve to further inform parametric models of solar heating of sea ice, particularly in light of recent results that emphasize the importance of daily forcing effects (Hanesiak and others, 1999). We plan to explore more fully the changes in deep amplitudes and layer thickness as ice temperature changes, in a future publication. We also plan to take advantage of small parameters that appear in the linearization Equation (14) and explore the implications of analytic asymptotic solutions for the oscillatory response of sea-ice temperature to penetrating solar radiation.

\section{ACKNOWLEDGEMENT}

We are grateful to S. Wilkinson for help with the 1999 data.

\section{REFERENCES}

Crocker, G. B. and P. Wadhams. 1989. Modelling Antarctic fast-ice growth. 7. Glaciol., 35(119), 3-8.

Eicken, H. 1992. Salinity profiles of Antarctic sea ice: field data and model results. F. Geophys. Res., 97 (C10), 15,545-15,557.

Flato, G. M. and R. D. Brown. 1996. Variability and climate sensitivity of landfast Arctic sea ice. 7. Geophys. Res., 101 (C11), 25,767-25,778.

Gow, A. J., S. F. Ackley, J.W. Govoni and W. F. Weeks. 1998. Physical and structural properties of land-fast sea ice in McMurdo Sound, Antarctica. In Antarctic Sea Ice: Physical Processes, Interactions and Variability, Antarctic Research Series 74, 355-374.

Grenfell, T. C. 1991. A radiative transfer model for sea ice with vertical structure variations. 7. Geophys. Res., 96(C9), 16,991-17,001.

Haines, E. M., R. G. Buckley and H. J. Trodahl. 1997. Determination of the depth dependent scattering coefficient in sea ice. 7. Geophys. Res., $102(\mathrm{Cl}), 1141-1151$.

Hanesiak, J. M., D. G. Barber and G. M. Flato. 1999. The role of diurnal processes in the seasonal evolution of sea ice and its snow cover. 7 . Geophys. Res., 104(C6), 13,593-13,603.

Jeffries, M. O., W. F. Weeks, R. Shaw and K. Morris. 1993. Structural characteristics of congelation and platelet ice and their role in the development of Antarctic land-fast sea ice. F. Glaciol., 39(132), 223-238.

Kevorkian, J. and J. D. Cole. 1981. Perturbation methods in applied mathematics. New York, Springer-Verlag. (Applied Mathematical Science 34.)

Key, J. R., R. A. Silcox and R. S. Stone. 1996. Evaluation of surface radiative flux parameterizations for use in sea ice models. 7. Geophys. Res., 101 (C2), 3839-3849.

McGuinness, M. J., H. J. Trodahl, K. Collins and T. G. Haskell. 1998. Nonlinear thermal transport and brine convection in first-year sea ice. Ann. Glaciol., 27, 471-476.

Ono, N. 1966. Thermal properties of sea ice. 3. On the specific heat of sea ice. Low Temp. Sci., Ser. A, 24, 249-258.

Perovich, D. K., C. S. Roesler and W. S. Pegau. 1998. Variability in Arctic sea ice optical properties. F. Geophys. Res., 103(Cl), 1193-1208.

Press, W. H., B. P. Flannery, S. A. Teukolsky and W.T. Vetterling. 1986. Numerical recipes: the art of scientific computing. Cambridge, Cambridge University Press.

Schwerdtfeger, P. 1963. The thermal properties of sea ice. F. Glaciol., 4(36), 789-807.

Smith, I. J., P. J. Langhorne, H. J. Trodahl, T. G. Haskell and D. M. Cole. 1999. Platelet ice - the McMurdo Sound debate. In Shen, H. T., ed. Ice in surface waters. Rotterdam, A. A. Balkema, 829-834.

Trodahl, H. J. and 6 others. 2000. Heat transport in McMurdo Sound firstyear fast ice. F. Geophys. Res., 105 (C5), 11,347-11,358.

Yen, Y.-C. 1981. Review of thermal properties of snow, ice and sea ice. CRREL Rep. 81-10.

Zeebe, R. E., H. Eicken, D. H. Robinson, D. Wolf-Gladrow and G. S. Dieckmann. 1996. Modelling the heating and melting of sea ice through light absorption by microalgae. 7. Geophys. Res., 101 (C1), 1163-1181. 